LMU-TPW-97/02

hep-th/9701047

\title{
The Coulomb potential in gauge theory with a dilaton
}

\author{
Rainer Dick \\ Sektion Physik der Universität München \\ Theresienstr. 37, 80333 München, Germany
}

\begin{abstract}
I calculate the potential of a pointlike particle carrying $\mathrm{SU}\left(N_{c}\right)$ charge in a gauge theory with a dilaton. The solution depends on boundary conditions imposed on the dilaton: For a dilaton that vanishes at infinity the resulting potential is of the form $\left(r+r_{\phi}\right)^{-1}$, with $r_{\phi}$ inverse proportional to the decay constant of the dilaton. Another natural constraint for the dilaton $\phi$ is independence of $\frac{1}{g^{2}} \exp \left(\frac{\phi}{f_{\phi}}\right)$ from the gauge coupling $g$. This requirement yields a potential proportional to $r$ and makes it impossible to create an isolated $\mathrm{SU}\left(N_{c}\right)$ charge.
\end{abstract}


1. Dilatons are scalar particles predicted by string theory and any theory involving a compactification scale [1]. An unambiguous property of a string or Kaluza-Klein dilaton $\phi$ in four dimensions besides its scalar transformation behaviour is its coupling to gauge fields through a term $\exp \left(\phi / f_{\phi}\right) F^{2}$, and I will use this as the defining property of a dilaton. Dilatons arise from the massless spectrum of fundamental strings in two different ways: On the one hand there is the model independent dilaton arising as a unique massless scalar state of closed superstrings, while on the other hand nonlinear combinations of ten-dimensional massless tensor states measure the volume of internal dimensions and appear as Kaluza-Klein dilatons in four dimensions. Linear combinations of these dilatons may couple in different ways to gauge fields arising from different sectors of string theory and from compactifications and the dilaton sector of low energy quantum field theories inherited from string theory can become quite complicated. In spite of these possible complications my main interest in the present paper concerns the discussion of a single low energy dilaton coupling to $\mathrm{SU}\left(N_{c}\right)$ gauge fields, in order to acquire a better understanding of the impact of dilatonic degrees of freedom in gauge theory.

Besides its appearance in the spectrum of string theory and Kaluza-Klein theories, the present investigation was also motivated by the fact that the dilaton begins to play an even more prominent rôle through its covariance under duality symmetries: Axion-dilaton-photon systems exhibit a non-linear duality symmetry mixing axions and dilatons through $\mathrm{SL}(2, \mathbb{R})$ transformations [2] and recent developments in string theory indicate that S-duality symmetries should be a generic feature of the kinetic sector of low energy quantum field theories [3].

It is familiar from the axion and apparent for the dilaton that a (pseudo-)scalar can be very light, yet very hard to observe if its decay constant is very large. A relevant problem then is the question how a light dilaton affects the Coulomb potential and its non-abelian analog. In order to study this problem we will neglect any dilaton mass in the sequel and study the dilaton-gluon field generated by a pointlike quark. For a dilaton vanishing at infinity we will find a modified Coulomb potential which is regularized at a radius

$$
r_{\phi}=\frac{g}{8 \pi f_{\phi}} \sqrt{\frac{1}{2}-\frac{1}{2 N_{c}}}
$$

for gauge group $\mathrm{SU}\left(N_{c}\right)$ and

$$
r_{\phi}=\frac{g}{8 \pi f_{\phi}}
$$

for $\mathrm{U}(1)$. On the other hand, a non-vanishing expectation value of the dilaton rescales the gauge coupling, giving rise to the requirement that $\exp \left(\frac{\phi}{f_{\phi}}\right)$ should scale like $g^{2}$.

\footnotetext{
${ }^{1}$ The Kaluza-Klein gauge fields disappear from the low energy theory if the compactification has a direct product structure globally.
} 
The unique solution satisfying this requirement yields a potential proportional to the distance $r$ from the source.

Throughout this paper I will use the language of QCD for gauge fields, charges and fermions. The translation of the results to an abelian gauge group is straightforward. I use letters from the middle of the alphabet both for spatial Minkowski space indices and colour indices, while letters from the beginning of the alphabet denote Lie algebra indices.

2. We are interested in $\mathrm{SU}\left(N_{c}\right)$ gauge theory augmented with a dilaton, and the theory is described by a Lagrange density

$$
\mathcal{L}=-\frac{1}{4} \exp \left(\frac{\phi}{f_{\phi}}\right) F_{\mu \nu}{ }^{a} F^{\mu \nu}{ }_{a}-\frac{1}{2} \partial^{\mu} \phi \cdot \partial_{\mu} \phi+\sum_{f=1}^{N_{f}} \bar{q}_{f}\left(i \gamma^{\mu} \partial_{\mu}+g \gamma^{\mu} A_{\mu}{ }^{a} X_{a}-m_{f}\right) q_{f},
$$

with $X_{a}$ denoting a defining $N_{c}$-dimensional representation of $\operatorname{su}\left(N_{c}\right)$.

This theory would arise e.g. through spontaneous compactification of five-dimensional QCD with $N_{f}$ quark flavours, see [四], with one exception: I did not include couplings of the dilaton to quark masses, since in the standard model masses are generated at the weak scale, far below any string or compactification scale. I also set the axion already to zero, since the static pointlike source considered below does not excite the axion field.

The equations of motion are

$$
\begin{gathered}
\partial_{\mu}\left(\exp \left(\frac{\phi}{f_{\phi}}\right) F^{\mu \nu}{ }_{a}\right)+g \exp \left(\frac{\phi}{f_{\phi}}\right) A_{\mu}{ }^{b} f_{a b}{ }^{c} F^{\mu \nu}{ }_{c}=-g \bar{q} \gamma^{\nu} X_{a} q, \\
\partial^{2} \phi=\frac{1}{4 f_{\phi}} \exp \left(\frac{\phi}{f_{\phi}}\right) F_{\mu \nu}{ }^{a} F^{\mu \nu}{ }_{a}, \\
\left(i \gamma^{\mu} \partial_{\mu}+g \gamma^{\mu} A_{\mu}{ }^{a} X_{a}-m\right) q=0,
\end{gathered}
$$

where here and in the sequel flavour indices are suppressed. To discuss the impact of the dilaton on the Coulomb potential we consider the Gauss law and Faraday's law for stationary configurations in the presence of a dilaton:

$$
\begin{gathered}
\nabla \cdot\left(\exp \left(\frac{\phi}{f_{\phi}}\right) \mathbf{E}\right)-i g \exp \left(\frac{\phi}{f_{\phi}}\right)(\mathbf{A} \cdot \mathbf{E}-\mathbf{E} \cdot \mathbf{A})=\varrho, \\
\nabla \times \mathbf{E}-i g(\mathbf{A} \times \mathbf{E}+\mathbf{E} \times \mathbf{A})=0,
\end{gathered}
$$

where in the gauge theory above $\varrho=g\left(q^{+} \cdot X_{a} \cdot q\right) X^{a}$ and $E_{j}=-F_{0 j}{ }^{a} X_{a}$.

The vector potential $\mathbf{A}$ is pure gauge in the static limit since the chromo-magnetic field

$$
\mathbf{B}=\nabla \times \mathbf{A}-i g \mathbf{A} \times \mathbf{A}
$$


vanishes, and we end up with

$$
\begin{gathered}
\nabla \cdot\left(\exp \left(\frac{\phi}{f_{\phi}}\right) \mathbf{E}\right)=\varrho, \\
\nabla \times \mathbf{E}=0
\end{gathered}
$$

Our aim is to determine the chromo-electric potential for a point charge

$$
\varrho_{a}(\mathbf{r})=g C_{a} \delta(\mathbf{r})
$$

where $C_{a}$ denotes the expectation value of the generator $X_{a}$ in colour space. From the relation

$$
\left(X_{a}\right)_{i j}\left(X^{a}\right)_{k l}=\frac{1}{2} \delta_{i l} \delta_{j k}-\frac{1}{2 N_{c}} \delta_{i j} \delta_{k l}
$$

one finds for arbitrary colour content

$$
\sum_{a=1}^{N_{c}^{2}-1} C_{a}^{2}=\frac{N_{c}-1}{2 N_{c}}
$$

We thus want to determine the field of a stationary pointlike quark from

$$
\begin{gathered}
\nabla \cdot\left(\exp \left(\frac{\phi(\mathbf{r})}{f_{\phi}}\right) \mathbf{E}_{a}(\mathbf{r})\right)=g C_{a} \delta(\mathbf{r}), \\
\nabla \times \mathbf{E}_{a}(\mathbf{r})=0
\end{gathered}
$$

and

$$
\Delta \phi(\mathbf{r})=-\frac{1}{2 f_{\phi}} \exp \left(\frac{\phi(\mathbf{r})}{f_{\phi}}\right) \mathbf{E}_{a}(\mathbf{r}) \cdot \mathbf{E}^{a}(\mathbf{r})
$$

The unique radially symmetric solution to (5) can be written down immediately:

$$
\exp \left(\frac{\phi(r)}{f_{\phi}}\right) \mathbf{E}_{a}(\mathbf{r})=\exp \left(\frac{\phi(r)}{f_{\phi}}\right) E_{a}(r) \mathbf{e}_{r}=\frac{g C_{a}}{4 \pi r^{2}} \mathbf{e}_{r}
$$

whence equation (6) is also satisfied. Equation (7) then translates into

$$
\frac{d^{2}}{d r^{2}} \phi(r)+\frac{2}{r} \frac{d}{d r} \phi(r)=-\frac{g^{2}}{64 \pi^{2} f_{\phi}}\left(1-\frac{1}{N_{c}}\right) \exp \left(-\frac{\phi(r)}{f_{\phi}}\right) \frac{1}{r^{4}}
$$

The form of this equation suggests an ansatz $\frac{\phi(r)}{f_{\phi}}=a \ln \left(\frac{r}{b}\right)$, which yields the solution discussed in the next section. However, we can solve (9) for arbitrary boundary conditions through a substitution

$$
\xi=\frac{g}{4 \pi f_{\phi} r} \sqrt{\frac{1}{2}-\frac{1}{2 N_{c}}}, \quad \psi(\xi)=\frac{\phi(r)}{f_{\phi}},
$$


yielding

$$
\frac{d^{2}}{d \xi^{2}} \psi(\xi)=-\frac{1}{2} \exp (-\psi(\xi))
$$

or in terms of boundary conditions at infinity:

$$
\begin{aligned}
& \psi^{\prime}(\xi)^{2}-\psi^{\prime}(0)^{2}=\exp (-\psi(\xi))-\exp (-\psi(0)), \\
& \xi=\int_{\psi(0)}^{\psi(\xi)} \frac{d \psi}{\sqrt{\exp (-\psi)-\exp (-\psi(0))+\psi^{\prime}(0)^{2}}},
\end{aligned}
$$

where a sign ambiguity has been resolved by the requirement that the dilaton should not diverge at finite radius. The integral can be done elementary, with two branches depending on the sign of $\psi^{\prime}(0)^{2}-\exp (-\psi(0))$.

The presence of the dilaton introduced a two-fold ambiguity in the Coulomb problem, and we have to determine from physical requirements which boundary conditions to chose.

Here we require that the dilaton generated by the pointlike quark vanishes at infinity while the gradient satisfies the minimality condition

$$
\lim _{r \rightarrow \infty} r^{2} \frac{d}{d r} \phi(r)=-\frac{g}{4 \pi} \sqrt{\frac{1}{2}-\frac{1}{2 N_{c}}} .
$$

This gives minimal kinetic energy for the dilaton at infinity subject to the constraint that the chromo-electric field does not develop a singularity for positive finite $r$. Then we find for the radial dependence of the dilaton and the electric field

$$
\begin{gathered}
\phi(r)=2 f_{\phi} \ln \left(1+\frac{g}{8 \pi f_{\phi} r} \sqrt{\frac{1}{2}-\frac{1}{2 N_{c}}}\right), \\
\mathbf{E}_{a}(\mathbf{r})=\frac{g C_{a}}{4 \pi\left(r+\frac{g}{8 \pi f_{\phi}} \sqrt{\frac{1}{2}-\frac{1}{2 N_{c}}}\right)^{2}} \mathbf{e}_{r},
\end{gathered}
$$

implying a modified Coulomb potential

$$
\Phi_{a}(r)=\frac{g C_{a}}{4 \pi r+\frac{g}{2 f_{\phi}} \sqrt{\frac{1}{2}-\frac{1}{2 N_{c}}}} .
$$

The result for gauge group $\mathrm{U}(1)$ is received through the substitution $N_{c} \rightarrow-1$.

This removal of the short distance singularity in the chromo-electric field would imply finite energy of the dilaton-gluon configuration:

$$
E=\int d^{3} \mathbf{r}\left(\frac{1}{2} \nabla \phi \cdot \nabla \phi+\frac{1}{2} \exp \left(\frac{\phi(\mathbf{r})}{f_{\phi}}\right) \mathbf{E}_{a}(\mathbf{r}) \cdot \mathbf{E}^{a}(\mathbf{r})\right)=2 g f_{\phi} \sqrt{\frac{1}{2}-\frac{1}{2 N_{c}}} .
$$


3. However, there exists another quite intriguing solution if we require that $\frac{1}{g^{2}} \exp \left(\frac{\phi}{f_{\phi}}\right)$ is independent of $g$. This requirement arises naturally in string theory, since the nonperturbatively fixed expectation value of the dilaton itself is supposed to determine the coupling. In the action (1) this requirement amounts to the constraint that the solution should respect the scale invariance of the equations of motion under

$$
\begin{gathered}
\phi \rightarrow \phi+2 \zeta f_{\phi} \\
A \rightarrow \exp (-\zeta) A \\
g \rightarrow \exp (\zeta) g
\end{gathered}
$$

for constant $\zeta$. Eqs. (10,12) then imply $\psi^{\prime}(\xi)^{2}=\exp (-\psi(\xi))=4 \xi^{-2}$, yielding

$$
\begin{gathered}
\phi(r)=2 f_{\phi} \ln \left(\frac{g}{8 \pi f_{\phi} r} \sqrt{\frac{1}{2}-\frac{1}{2 N_{c}}}\right), \\
\mathbf{E}_{a}(\mathbf{r})=\frac{32 \pi f_{\phi}^{2}}{g} \frac{N_{c}}{N_{c}-1} C_{a} \mathbf{e}_{r} .
\end{gathered}
$$

This corresponds to an energy density

$$
\mathcal{H}(\mathbf{r})=4 \frac{f_{\phi}^{2}}{r^{2}}
$$

whence the energy in a volume of radius $r$ diverges linearly:

$$
\left.E\right|_{r}=16 \pi f_{\phi}^{2} r .
$$

This is an infrared divergence which we cannot expect to be related to new physics at short distances, and it would cost an infinite amount of energy to create an isolated quark.

Note added: The abelian version of the solution (14)-(16) was introduced as a solitonic excitation of the dilaton-photon system in a remarkable paper by Cvetič and Tseytlin [5]. I would like to thank A. Tseytlin for drawing my attention to this work.

\section{References}

[1] M.B. Green, J.H. Schwarz and E. Witten, Superstring Theory, 2 Vols., Cambridge University Press, Cambridge 1987.

[2] A. Shapere, S. Trivedi and F. Wilczek, Mod. Phys. Lett. A6 (1991) 2677. 
[3] A. Font, L. Ibañez, D. Lüst and F. Quevedo, Phys. Lett. B249 (1990) 35; J.H. Schwarz and A. Sen, Nucl. Phys. B411 (1994) 35, Phys. Lett. B312 (1993) 105 ;

A. Sen, Int. J. Mod. Phys. A9 (1994) 3707, Phys. Lett. B329 (1994) 217;

J.H. Schwarz, Lectures on superstring and $M$ theory dualities, hep-th/9607201.

[4] R. Dick, Phys. Lett. B380 (1996) 29.

[5] M. Cvetič and A.A. Tseytlin, Nucl. Phys. B416 (1994) 137. 